\title{
Erratum to: The Quantum Steganography Protocol via Quantum Noisy Channels
}

\author{
Zhan-Hong Wei ${ }^{1,2} \cdot$ Xiu-Bo Chen ${ }^{1,2} \cdot$ Xin-Xin Niu ${ }^{1,2}$. \\ Yi-Xian Yang ${ }^{1,2}$
}

Published online: 4 June 2015

(C) Springer Science+Business Media New York 2015

\section{Erratum to: Int J Theor Phys (2014) \\ DOI 10.1007/s10773-014-2478-x}

The original version of this article unfortunately contained a mistake. Figure 1 in the original publication is incorrect. The correct version of Figure 1 is shown below:

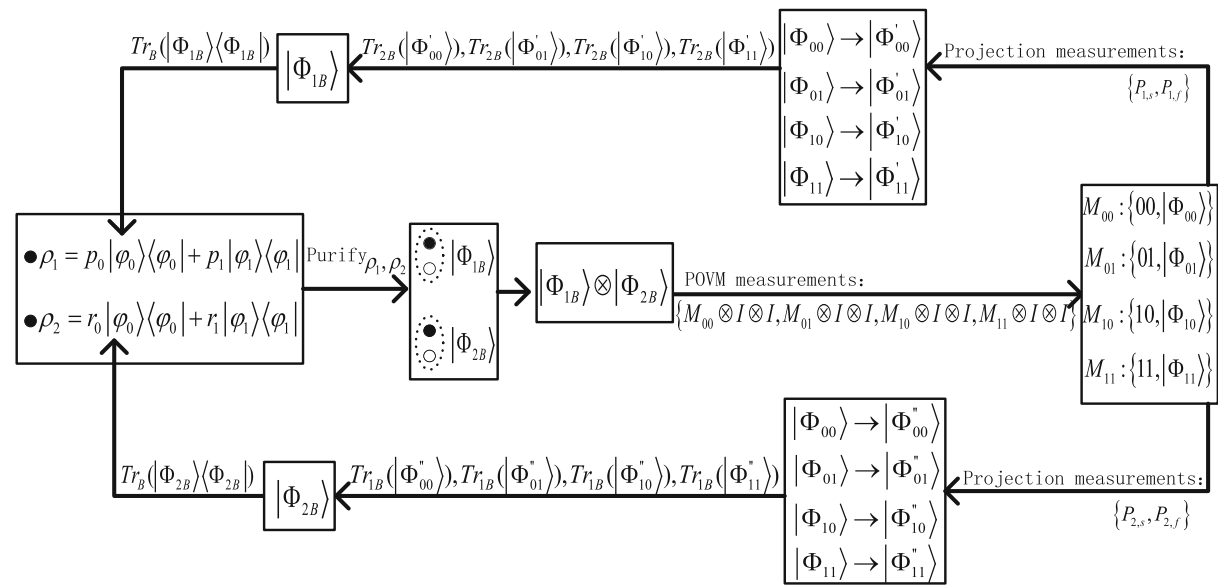

The online version of the original article can be found at http://dx.doi.org/10.1007/s10773-014-2478-x.

Xiu-Bo Chen

flyover100@163.com

Zhan-Hong Wei

wwwzhh-85@163.com

1 Information Security Center, State Key Laboratory of Networking and Switching Technology, Beijing University of Posts and Telecommunications, Beijing 100876, China

2 State Key Laboratory of Information Security, Institute of Information Engineering, Chinese Academy of Sciences, Beijing 100093, China 